Letter to the Editor

\title{
Bilateral posterior capsule injury after Nd:YAG laser vitreolysis: unintended consequence of floaters treatment
}

\author{
Roderick O'Day MBBS, ${ }^{1,2}$ Dean Cugley $\mathrm{MBBS}^{1}{ }^{1}$ Christine Chen PhD FRANZCO ${ }^{2}$ and \\ David Fabinyi FRANZCO ${ }^{1}$
}
${ }^{1}$ Royal Victorian Eye and Ear Hospital, 32 Gisborne Street, East Melbourne, Vic 3002, Australia
${ }^{2}$ Department of Surgery, Clinical Sciences at Monash Health, Monash University, 246 Clayton Road, Clayton, Vic 3168, Australia

Correspondence: Roderick O’Day, Royal Victorian Eye and Ear Hospital, 32 Gisborne Street, East Melbourne, Vic 3002, Australia

Email: roderick.oday@gmail.com

Received 5 March 2018; accepted 8 March 2018

Conflict of interest: None

Funding sources: None

This is the author manuscript accepted for publication and has undergone full peer review but has not been through the copyediting, typesetting, pagination and proofreading process, which may lead to differences between this version and the Version of Record. Please cite this article as doi: 10.1111/ceo.13190

This article is protected by copyright. All rights reserved. 
Patients with symptomatic vitreous floaters are common in clinical practice.(1) Current management options include observation, pars plana vitrectomy ('PPV') or Nd:YAG laser vitreolysis. Most patients are managed conservatively with reassurance and an explanation of the cause of their symptoms. Some, however, find the symptoms debilitating. Nd:YAG laser vitreolysis has been proposed as a potential treatment for these patients. Proponents of this treatment argue that in certain patients it may be safer than PPV. There is no risk of endophthalmitis and theoretically less risk of cataract formation. $(1,2)$ Perhaps due to limited literature on its use, Nd:YAG laser vitreolysis has been reported to have a good side effect profile, with only uveitis and raised intraocular pressure occurring commonly.(1) Nd:YAG laser can, however, cause significant damage to intraocular structures. We describe a case of bilateral cataracts with posterior capsule defects caused by Nd:YAG laser vitreolysis.

A 46-year-old female was referred directly from private rooms for left cataract surgery at a public hospital. The patient had complained to the private specialist of significant floaters in both eyes, which had been unsuccessfully treated with bilateral Nd:YAG laser vitreolysis. The patient was found to have posterior subcapsular cataracts ('PSCC') and left cataract surgery was arranged. Posterior capsule defects were not identified by the specialist prior to the left cataract surgery. Pre-operative best-corrected visual acuity ('BCVA') was 6/9 in both eyes. The cataract surgery was complicated by partial nucleus drop after a posterior capsule defect was encountered on removal of the first nuclear segment. An anterior vitrectomy was performed, residual nucleus and cortex removed and sulcus fixated posterior chamber intraocular lens ('PCIOL') inserted with optic capture. Two weeks postoperatively, BCVA was $6 / 9$ in the right eye and 6/6 in the left eye. The right anterior segment showed superotemporal PSCC with a localised posterior capsule defect. (Figures 1 \& 2) Right posterior segment examination was unremarkable. Examination of the left 
eye showed a clear cornea, minimal intraocular inflammation, the PCIOL and three small nucleus fragments in the inferior vitreous. The patient underwent uncomplicated left PPV one week later. Six weeks post-PPV, BCVA was $6 / 9$ in both eyes. There have been no further complications. Right cataract surgery was postponed until the vision deteriorates further.

It is likely that Nd:YAG laser vitreolysis caused cataract formation with posterior capsule defects in our patient. This complication has been recently reported in three patients. $(3,4)$ It is not clear if the mechanism of this complication is inadvertent focusing of the laser beam adjacent to the posterior capsule or if the cataract and posterior capsule defect are caused by another mechanism. Our case highlights the importance in clinicians being aware of this complication. The posterior capsule defect was unrecognised prior to cataract surgery. Had it been, preoperative planning and vitreoretinal involvement may have obviated the need for a second surgery.

The side effect profile of Nd:YAG laser vitreolysis is not fully known. Most initial reports of this treatment for symptomatic vitreous floaters were limited case series or case reports. Recently, a randomised-controlled trial of Nd:YAG laser vitreolysis for the treatment of Weiss ring induced floaters was published.(5) No adverse events were encountered in the 36 eyes that underwent Nd:YAG laser vitreolysis in this study, although it was inadequately powered to assess for uncommon adverse events.(5) Nd-YAG laser vitreolysis is not necessarily a simple procedure. An average Nd:YAG laser vitreolysis treatment can consist of 218 shots with a mean total power of $1316 \mathrm{~mJ}$.(5) When used in the vitreous for alternate indications, Nd;YAG laser may cause retinal holes, vitreous haemorrhage, chorioretinal bleeding and elevated intraocular pressure.(1) Caution should be exercised when interpreting the side effect profile of this treatment from the limited published data of its use, given the 
difficulty of the procedure and the incidence of complications when Nd:YAG laser is used within the vitreous cavity for other indications.

Our case highlights a newly described complication of Nd:YAG laser vitreolysis treatment and the importance in recognising it preoperatively. With new RCT evidence of the potential benefit of Nd:YAG laser vitreolysis, this treatment may become more widely adopted. If so, further studies should performed to more accurately assess its side effect profile. 


\section{REFERENCES}

1. Milston $R$, Madigan $M C$, Sebag J. Vitreous floaters: Etiology, diagnostics, and management. Surv Ophthalmol 2016; 61: 211-27.

2. Delaney YM, Oyinloye A, Benjamin L. Nd:YAG vitreolysis and pars plana vitrectomy: surgical treatment for vitreous floaters. Eye (Lond Engl) 2002; 16: 21-6.

3. Sun $\mathrm{I}-\mathrm{T}$, Lee $\mathrm{T}-\mathrm{H}$, Chen $\mathrm{C}-\mathrm{H}$. Rapid Cataract Progression after Nd:YAG Vitreolysis for Vitreous Floaters: A Case Report and Literature Review. Case Rep Ophthalmol. 2017; 8: 321-5.

4. Koo EH, Haddock $L$, Bhardwaj N, Fortun JA. Cataracts induced by neodymium-yttrium-aluminium-garnet laser lysis of vitreous floaters. $\mathrm{Br} J$ Ophthalmol. 2017; 101: 709-11.

5. Shah CP, Heier JS. YAG Laser Vitreolysis vs Sham YAG Vitreolysis for Symptomatic Vitreous Floaters: A Randomized Clinical Trial. JAMA Ophthalmol. 2017;135: 918-23.

\section{FIGURE LEGENDS}

Figure 1: Slit-lamp photography of the right eye on retroillumination showing superotemporal cataract with posterior capsule defect

Figure 2: Slit-lamp photography of the right eye showing superotemporal cataract 


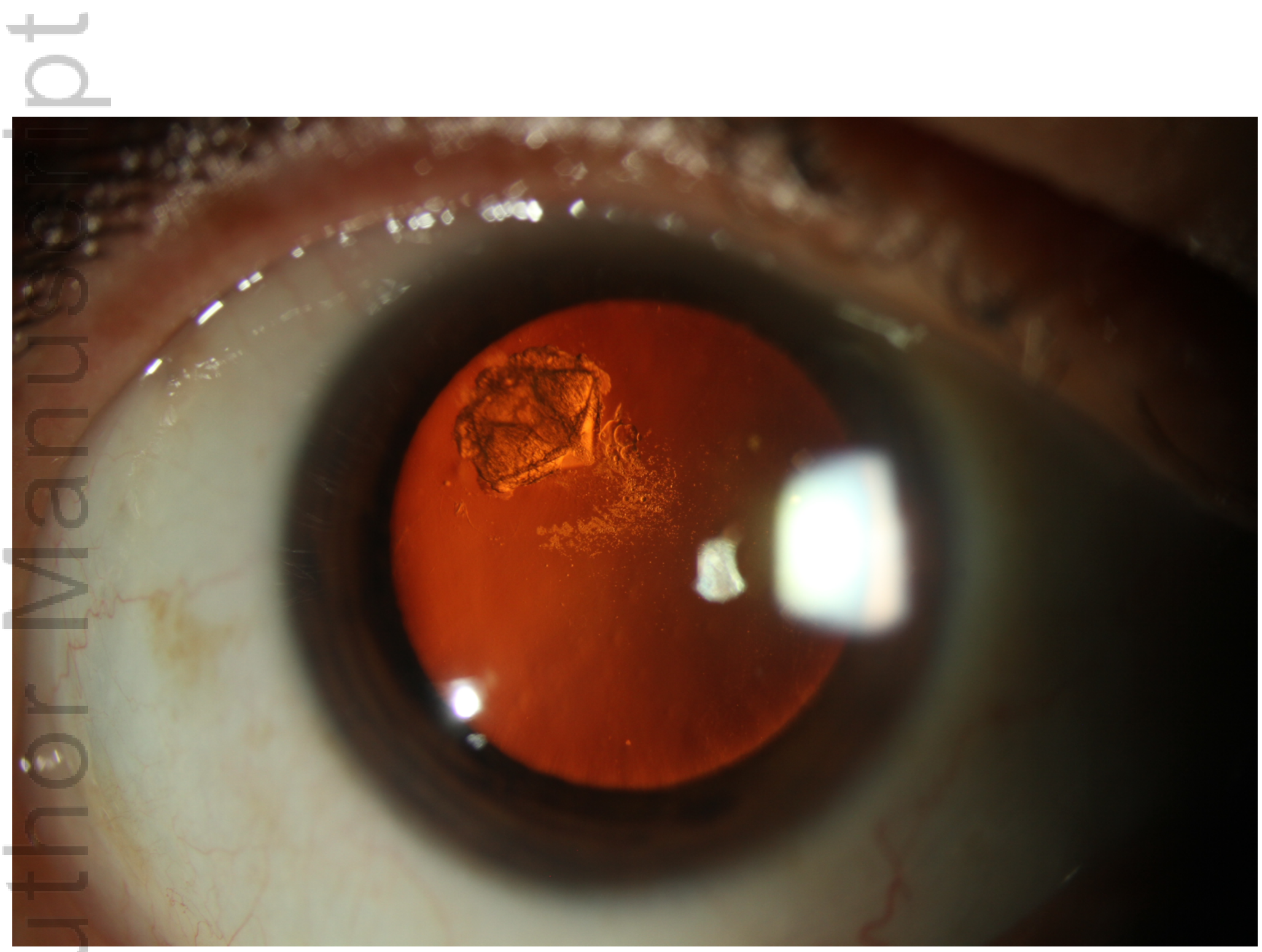

Figure 1.tif

This article is protected by copyright. All rights reserved. 

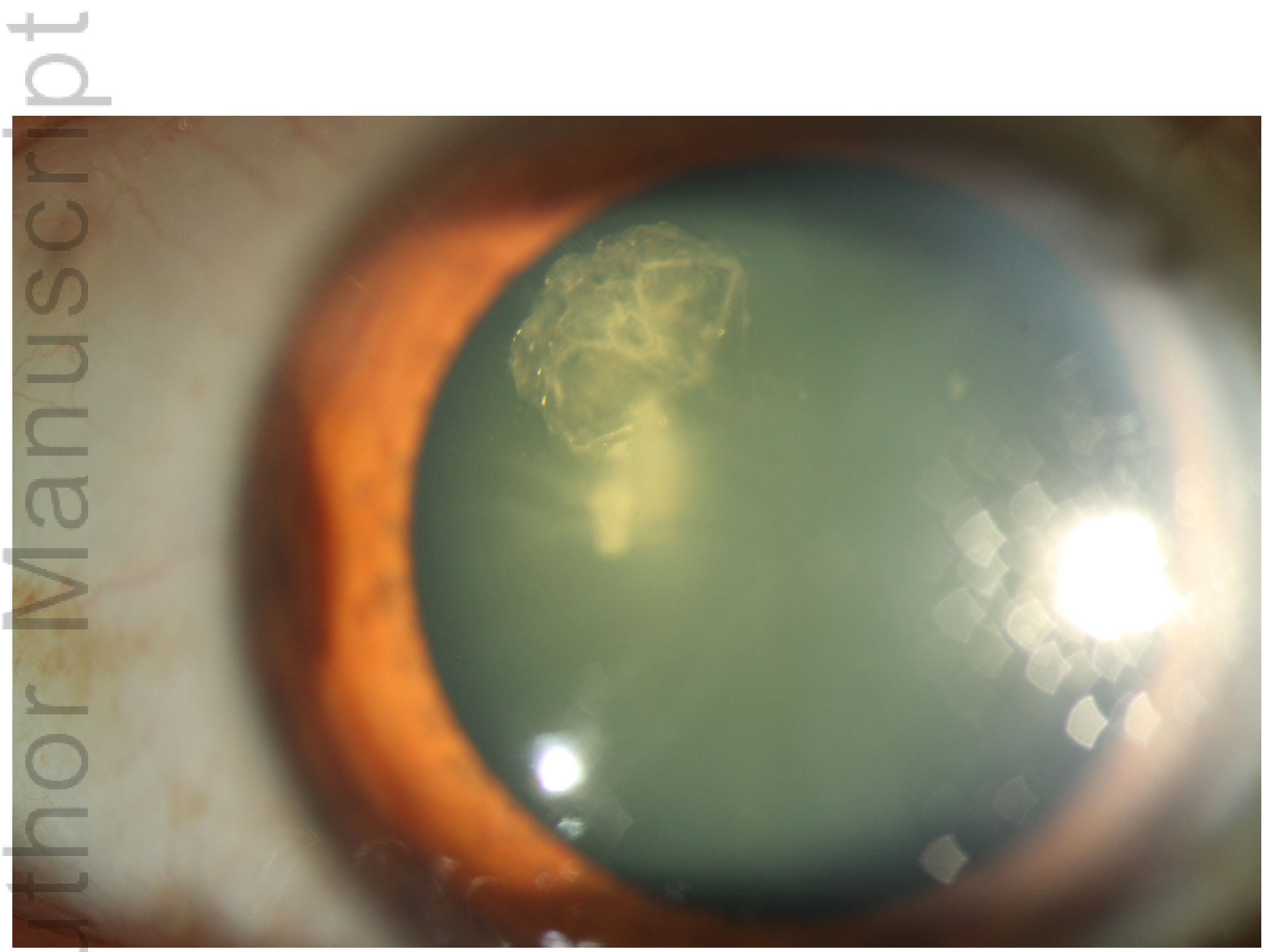

Figure 2.tif

This article is protected by copyright. All rights reserved. 